Historic, Archive Document

Do not assume content reflects current scientific knowledge, policies, or practices. 



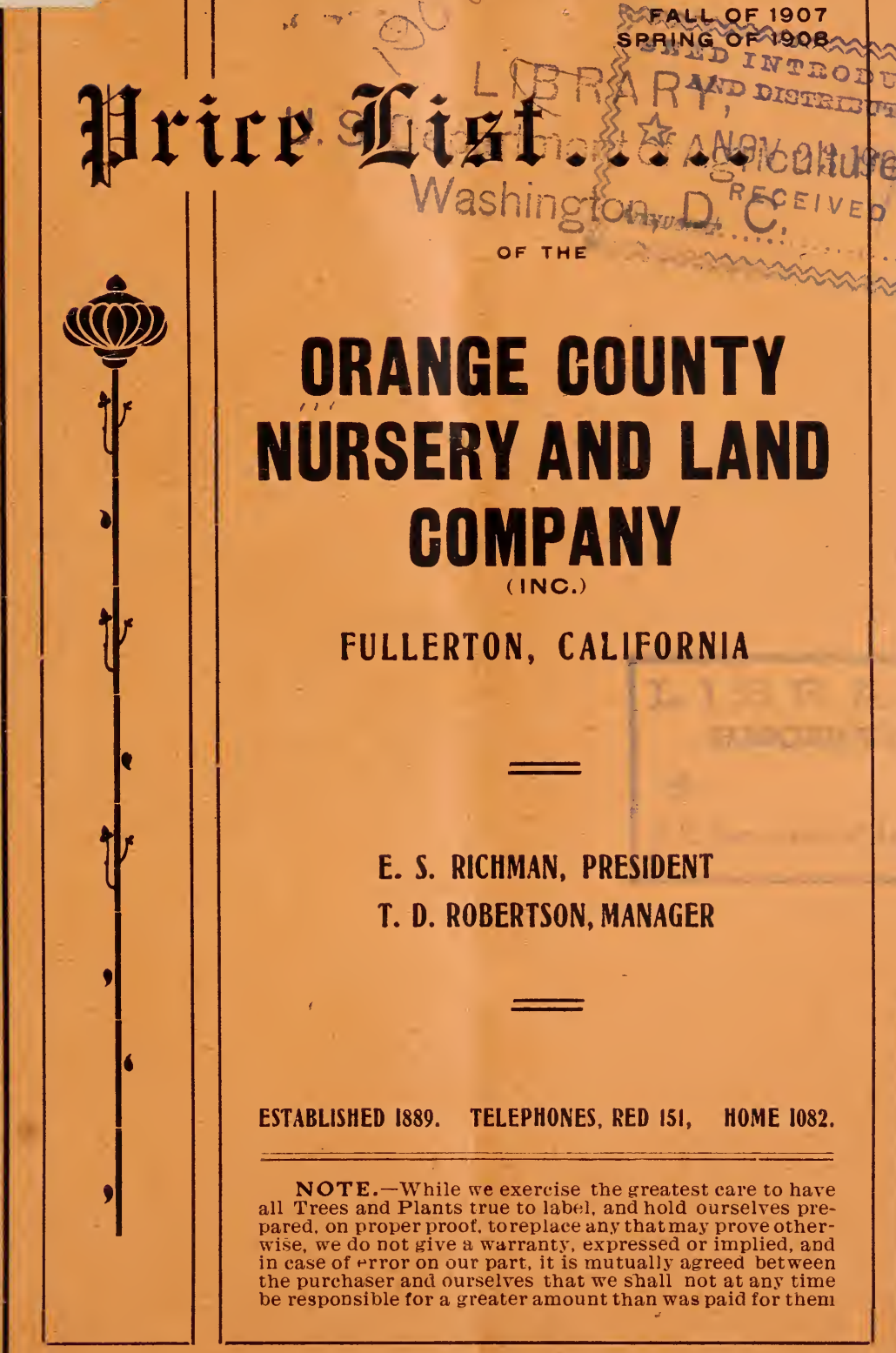




\section{INSTRUCTIONS TO PLANTERS.}

Order your trees early. Those who order first will be served first. If you delay your order until late in the season, you may not get what you want.

Deciduous trees should be planted as soon as possible after the leaves liare fallen, and the ground can be gotten into good condition.

Open the bundle or box as soon as received, and "heel in' in good, moist soil.

In planting. be sure and dig holes deep enough and large enough to admit all the roots withont bending or crowding. Fill in around the roots with well pulverized surface soil, packing firmly, except on top.

If the condition of the soil is such as to require the use of water at the time of planting, if the ground camnot be irrigated, the water should be poured into the hole after it is partly filled with earth. Allow the water to soak away, and then fill in with loose earth without packing.

Those who desire further instructions in regard to planting and care of plants, we would recommend to purchase a copy of Prof. E. J. Wickson's book, "California Fruits and IIow to Grow them."

How to Peach Our Nurseries.-Fullerton is on the Santa Fe Railroad: There are five passenger trains each day from the north via Los Angeles and five from the south via Orange.

The office, retail yards and ornamental department are located on West Commonwealth Ave:, one-half mile west of the First National Bank. The deciduous fruit and nut tree nurseries are located at the extreme west end of Commonwealth Are., 21/2 miles west of the ornamental nurseries and office.

Those contemplating making purchases will find it to their advantage to see our stock before buying.

Visitor's are always welconie. 


\section{PRICE LIST \\ of rit}

\section{ORANGE COUNTY NURSERY \& LAND COMPANY}

FULLERTON, CALIFORNIA.

\section{TERIIS AND CONDITIONS.}

First-Parties unknown to us must accompany their orders with cash or satisfacțory reference.

Second-Special prices will be given on large specified orders.

Third-We pack in the best possible manner. either by covering with tules or in boxes, thus making a saving of onethird in freight charges. A nominal charge. to cover actual cost of packing, will be made.

Fourth-REMITTANCES. Remittances should be sent by check, postoffice order, express money order, or bank draft.

Fifth-In ordering, state whether substitution will be allowed or not. It frequently happens in the latter part of the season that we are out of certain varieties. If not specially requested not to substitute, we reserve the privilege of doing so, when it can be done without disadvantage to the purchaser. In fact, purchasers of stock for a home orchard or home grounds, if not specially conversant with varieties will. do well to leave the entire selection to us, as we will send only such varieties as are suited to the locality.

Sixth-Cut flowers and all kinds of floral work by express. These orders must be accompanied by the cash. We guarantee satisfaction in all our floral work:

Seventh-We all make mistakes. We will cheerfully rectify any that are brought to our notice if complaint is promptly made. 


\section{NOTE}

Our stock of Fruit Trees is very fine, being well grown and clean stock. Three hundred or more trees of one variety go at 1000 rate; thirty or more at 100 rate; five or more at 10 rate; less than five of one variety at the single rate.

Apple-One year old.

Fancy

Choice

Varieties: low Transparent, Red June, Maiden's Blush, Fameuse (Snow Apple), Yellow Belleflower, Gravenstein, Jonathan, R. I. Greening, Ben Davis, Spitzenberg, Smith's Cider, Rome Beauty, Yellow Newton Pippin, Missouri Pippin, Northern Spy, W. W. Pearmain, Arkansas Beauty, Arkansas Black, Mammoth Black Twig, Wine Sap, Delaware Red Winter (Lawver), Baldwin, White Pippin, Duchess of Oldenberg, Grime's Golden, Tollman Sweet, Fall Pippin, Wealthy, York Imperial, Gano, Summer Queen.

Crab Apple-One year old.

Fancy -.....

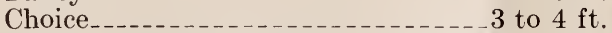

Varieties: Yellow Siberian, Transcendent, Red Siberian, Florence, Whitney and Hyslop.

\section{Pear-}

4 to $6 \mathrm{ft}$

3 to $4 \mathrm{ft}$.

Varieties in Order of Ripening: Sugar Pear, Bartlett, Clapp's, Favorite, Duchess, Seckel Beurre d'Anjou, Kieffer, Flemish Beauty, Winter Nelis.

Pomegranate-20 to 35 cents each.

Quince-15 to 25 cents each.

\section{Cherry-}

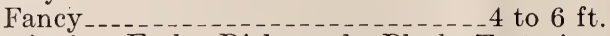

Varieteis: Early Richmond, Black Tartarian.

\section{Apricot-}

एकास

On Peach, one year

Choice $-\frac{1}{2}$ to $\frac{3}{4}$ inch...

Standard

Varieties: Ey. Golden, Royal, Moorpark, Newcastle, Tilton.

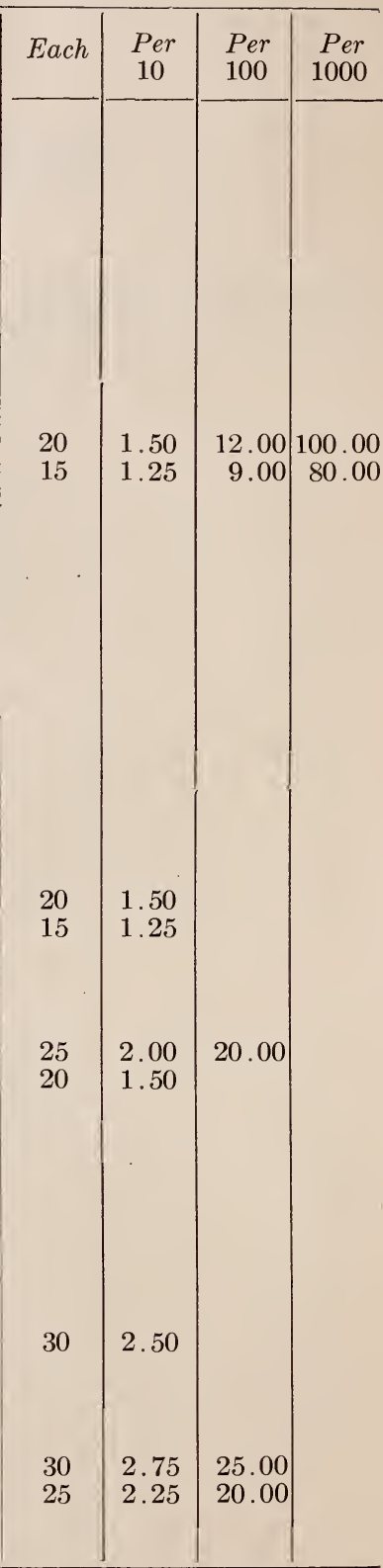


Peach-On Peach. One year old.

Choice $-\frac{1}{2}$ to $\frac{3}{4}$ in. Standard

Freestones in Order of ripening: Australian Saucer, Japanese Dwarf, Brigg's Red May, Alexander, Imperial, Early St. John, Carman Pallas, Honey, Foster, Crawford's Early, Muir, Lovell, Elberta, Wheatland, Late Crawford, Susquehanna, Wonderful, Salway.

Muir and Lovell $2 \mathrm{c}$ higher than other varieties.

Clingstones: Indian Cling, Lemon Cling, Orange Cling, Chinese Cling, Heath Cling, Henrietta Cling.

Tuscan and Phillip's Cling

Plum-On Peach.

Choice $-\frac{1}{2}$ to $\frac{3}{4}$ in. Standard _..................... 3 to $4 \mathrm{ft}$.

Varieties: Burbank, Kelsey, Satsuma, (blood), Wickson, Hale, Imperial Gage, Blue Damson, Yellow Egg, Gonzales.

Prune-On Peach.

Choice. 4 to $6 \mathrm{ft}$.

Tarieties: French, Imperial, Sugar, Tragedy, German, Italian.

Nectarine-On Peach. One Year.

New White, Boston, Jumbo.

Choice $-\frac{1}{2}$ to $\frac{3}{\frac{1}{4}}$ in................

Figs -3 to $4 \mathrm{ft}$.

\begin{tabular}{c|c|c|c} 
Each & $\begin{array}{c}\text { Per } \\
10\end{array}$ & $\begin{array}{c}\text { Per } \\
100\end{array}$ & $\begin{array}{c}\text { Per } \\
1000\end{array}$ \\
\hline & & & \\
25 & 2.25 & 20.00 & \\
20 & 1.75 & 16.00 &
\end{tabular}

2 to $3 \mathrm{ft}$.

Tarieties: Black Mission, Black Spanish,

Brown Turkey, White Adriatic, White Smyrna, and Calimyrna.

\section{Loquats-}

Budded Advance.............. 4 to $5 \mathrm{ft}$.

Budded Advance.................. 3 to $4 \mathrm{ft}$.

Budded Advance.................... $2 \mathrm{ft}$.

Seedlings _...........

Guava- (Strawberry) Pot Plants.

10 to 25 cents each; 75 c to -2.50 per dozen.

Guava-(Lemon) ................ 12 to 18 in.

Persimmons - (Japanese) -........... 3 to $4 \mathrm{ft}$.

Grapes

Varieties: Flame Tokay, Mission, White Muscat, Thompson's Seedless, Concord, Red Emperor, Cornichon, Rose of Peru, Black Prince and Zinfandel.

Write for prices on large orders.

Strawberry Plants-Arizona EverbearingImproved Noble Brandywine,

Dewberry-Gardena Signal Hill Advance Dewberry (very early) - $\$ 2.00$ per doz. 
Raspberries - Cuthbert

Blackberry - Wilson, Mommoth.

Loganberry

Currant-Red, White

Gooseberry

Olive-IV rite for Prices on Olives.

\section{FRUITS}

Oranges $-\frac{5}{8}$ and up

Varieties: Washington Navel, St. Michael, Mediterranean Sweet, Tangerine, Satsuma or Oonshiu Japanese, Valencia Late.

Oranges- Same as above but $\frac{1}{2}$ to $\frac{5}{8}-$

Lemons -3 to $4 \mathrm{ft}$.

Varieties: Villa Franca and Eureka.

Grape Fruit-3 to 4 feet

Variety: Marsh's Seedless.

Kum Quat-3 to $4 \mathrm{ft}$

Lime -3 to $4 \mathrm{ft}$.

\section{NUTS}

Almond-On Peach. One year.

Fancy

4 to $6 \mathrm{ft}$.

Choice

3 to $4 \mathrm{ft}$.

Varieties: I X L, Nonpariel, Ne Plus Ultra, Sultana.

Walnut-Santa Barhara (soft shell).

7 to $8 \mathrm{ft}$

6 to $7 \mathrm{ft}$

5 to $6 \mathrm{ft}$

4 to $5 \mathrm{ft}$

3 to $4 \mathrm{ft}$

2 to $3 \mathrm{ft}$

Walnut-Grafted on Black Walnut root.

(Placentia Perfection).

4 to $5 \mathrm{ft}$.

5 to $6 \mathrm{ft}$

6 to $9 \mathrm{ft}$.

8 to $12 \mathrm{ft}$

t-

\section{Chestnuts-}

3 to $5 \mathrm{ft}$.

Pecans-

2 feet

\begin{tabular}{|c|c|c|c|}
\hline Each & $\begin{array}{l}\text { Per } \\
10\end{array}$ & $\begin{array}{l}\text { Per } \\
100\end{array}$ & $\begin{array}{c}P e r \\
1000\end{array}$ \\
\hline & $\begin{array}{l}.40 \\
.40\end{array}$ & $\begin{array}{l}1.00 \\
1.00\end{array}$ & \\
\hline & .50 & 3.00 & 25.00 \\
\hline .15 & .90 & & \\
\hline 1.50 & 14.00 & 125.00 & \\
\hline $\begin{array}{l}1.25 \\
1.50\end{array}$ & 10.00 & & \\
\hline 1.50 & & & \\
\hline $\begin{array}{l}1.00 \\
1.00\end{array}$ & & & \\
\hline .30 & 2.75 & 25.00 & \\
\hline .25 & 2.00 & 20.00 & \\
\hline .40 & 4.00 & 37.50 & \\
\hline .35 & 3.50 & 32.50 & \\
\hline .30 & 3.00 & 27.50 & \\
\hline .25 & 2.50 & 24.00 & \\
\hline .25 & 2.25 & 20.00 & \\
\hline .20 & 1.75 & 15.00 & \\
\hline 60 & 5.50 & 50.00 & \\
\hline .75 & 7.00 & 60.00 & \\
\hline 1.00 & 9.00 & 70.00 & \\
\hline 1.50 & 13.00 & 100.00 & \\
\hline .35 & 3.00 & & \\
\hline 35 & & & \\
\hline
\end{tabular}




\section{EVERGREEN TREES.}

Acacia-Baileyana._.

Baileyana Baileyana Cultraformis. Dealbata. Decurrens. Decurrens... Floribunda.. Floribunda. Linafolia... Melanoxylon Melanoxylon. Melanoxylon Iollissima Mollissima Pycnantha Pycnantha Pycnantha Saligna Verticillata...

\begin{tabular}{|l|l} 
Each & Per \\
.40 & 3.50 \\
.60 & 5.50 \\
.75 & 7.00 \\
.35 & \\
.40 & 3.50 \\
.30 & 2.50 \\
.40 & 3.50 \\
.40 & 3.50 \\
.50 & 4.50 \\
.25 & \\
.40 & 3.50 \\
.50 & 4.50 \\
.75 & 7.00 \\
.40 & 3.50 \\
.50 & \\
.30 & 2.50 \\
.45 & 4.00 \\
.50 & \\
.25 & 2.00
\end{tabular}

Brachychiton-(See Sterculia).

Camphor._. Casuarina (Beefwood)

Casuarina stricta Chermoia-(Custard apple) Eucalyptus - Sugar gum_._._._._._._. 2 to $3 \mathrm{ft}$. in cans Sugar gum Sugar gum. Citradora Ficifolia, (scarlet flowering)

Leucoxylon

Polyanthema Robusta Robusta Rostrata, red gum cans Rostrata, red gum Rudis..... in cans Rudis.... Globulus, blue gum Sideroxylon

Ficus -Elastica_....... 12 to 18 in. in cans Australis.... .30 Australis Macrophylla.. Grevillea Robusta (Australian silk oak 2 to $3 \mathrm{ft}$.______in cans .20 Jacaranda Mimosifolia._._. .50 Hakea Eucalyptoides. Lagunaria Pattersoni Laurus Nobilis (Sweet Bay tree) ) Laurus Nobilis (Sweet Bay tree)_...........2 to $3 \mathrm{ft}$. in cans .35 Laurus Nobilis (Sweet Bay tree) _..._._._. 3 to $4 \mathrm{ft}$. in cans .50 Laurus Nobilis (Sweet Bay tree) in tubs, stems 40 to 48 in., Tops, 24 to 28 in. Tops, 28 to 30 in. 30.00 Tops, 30 to 34 in. 
Live Oak 8 to 12 in. in cans

Live Oak 12 to 18 in. in cans

Live Oak 18 to $24 \mathrm{in.}$ in cans Ligustrum-Gigantea Magnolia-Grandiflora_._._._._._. in pots 10 to 15 in. Magnolia-Grandiflora_..._._. Pepper Trees. Pepper Trees 2 to $3 \mathrm{ft}$. in cans

Pepper Trees. 3 to $4 \mathrm{ft}$. bare roots

Pittosporum undulatum, 1 to $2 \mathrm{ft}$. $35 \mathrm{c}$.

Sterculia-Diversifolia___._. Sterculia-Diversifolia_............ Sterculia-Diversifolia _............ Sterculia-Diversifolia_.......... 2 to $3 \mathrm{ft}$. balled and sacked Sterculia-Diversifolia_......... 3 to $4 \mathrm{ft}$. balled and sacked Sterculia-Diversifolia 4 to $5 \mathrm{ft}$. balled and sacked .90 Sterculia-Diversifolia 5 to $6 \mathrm{ft}$. balled and sacked 1.25 Sterculia-Diversifolia 6 to $8 \mathrm{ft}$. balled and sacked 2.00

Araucaria-Bidwilli_._._._. 14 to 14 in pots 2.00 Bidwilli Compacta__. Excelsa Excelsa Excelsa Glauca Cedrus deodora Deodora _._._. balled 1.50 Atlantica_._._. Libanus _......... 35

Cryptomera-Elegans Japonica _._. $2 \mathrm{ft}$. in cans .40

Cypress-Guadaloupe._._._._.

Italian pillar _...

Monterey _._._.

Monterey _...

Pinus-Insignis_._._. cans Insignis__._.

Insigni $3 \mathrm{ft}$. in cans

Insignis, balled and sacked, 4 to $5 \mathrm{ft}$

Canariensis

Canariensis

$1 \mathrm{ft}$. in cans

Parryana

$2 \mathrm{ft}$. in cans

Rosedale - Hybrid 1 to $2 \mathrm{ft}$. in cans .20

Sequoia-Gigantea $1 \mathrm{ft}$. in pots .50 . $1 \mathrm{ft}$. in 5 in. pots .40 Gigantea Sempervirens (Redwood) _._._._._. 1 to $2 \mathrm{ft}$. in 5 in. pots .35 Taxus erecta pyramidalis_................. 1 to $2 \mathrm{ft}$. 60 Thuja Aurea _._. Thuja Aurea Thuja Aurea-Common Arbor Vitae Thuyopsis dolobrata 


\section{DECIDUOUS TREES.}

Ash-White

Each

Elm-(Ulmus Americana)

Cork Elm

5 to

English

$8 \mathrm{ft} . \quad .25$

Catalpa

2 to

$3 \mathrm{ft}$.

8 to $10 \mathrm{ft}$.

Kaempferri

8 to $10 \mathrm{ft}$.

Bignanoides

8 to $10 \mathrm{ft}$.

Box Elder

8 to $10 \mathrm{ft}$

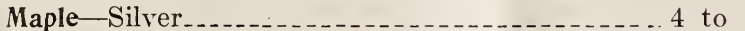

Maple-Silver._._._._.

Tulip Poplar

$6 \mathrm{ft}$.

$8 \mathrm{ft}$.

Walnut-Eastern Black.

$3 \mathrm{ft}$.

Texas Umbrella.

4 to

Texas Umbrella

Per

10

2.00

1.90

2.00

1.00

2.00

.25

25

.25

.25

2.00

2.00

2.00

4.00

5.50

\section{EVERGREEN SHRUBS.}

Abutilon.

Tarieties: Thompsonii (double yellow variety), Golden Bells (single golden yellow), Souv. de Bonne, Silver Leaf (rèd flower) Rosaflora (red).

Camellias-(Japonica) different colors $75 \mathrm{c}$ to -1.50 each. Cocoloba... Cocoloba Euphorbia-Splendens, Crown of Thorns Fuchsias. . Varieties: Luster, Elm City, Phenominal, Avalanche, Chas. Blanc, White Phenominal, Mrs. E. G. Hill, Black Prince, Earl of Beaconsfield, Storm King, Speciosa, Col. Roberts.

Hibiscus-In the following varieties. Single crimson.

Double crimson and Peachblow........ 6 to 12 in. plants

Double crimson and Peachblow. 12 to 18 in. plants

Hibiscus-Crimson Eye (White Hibiscus with crimson center) 1.00

Hibiscus_Manihot (Yellow Japanese) _.........2 to $3 \mathrm{ft} .1 .00$

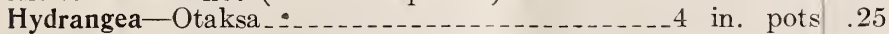

Thomas Hogg.

Varieties: Weeping (lavander), Grand Sultan (orange red), Alba Perfecta (white), Countess Brencourt (pink), Countess Riza du Parc (yellow and pink).

Streptosolon Jamesonii.

\section{DECIDUOUS SHRUBS.}

Bird of Paradise

Bridal Wreath

Crape Myrtle-pink

Crape Myrtle-Crimson

Crape Myrtle-White 
Poinsettias

Snow Ball.

Lilac-Purple

Lilac-White

\section{Herbaceous, Ornamental and Flowering Plants.}

Asparagus-Plumosus Nanus_._._._. Plumosus Nanus__._._._._. pots Sprengeri_____. pots Sprengeri-_._. Hanging Baskets..........

Banana-(fruit bearing) _._._._.

Muse Ensete

Begonias-in the following varieties: Rubra, Robusta, Edmondsii, Thurstonii, Sandersonii, McBethii, Madam de Lesseps, Weltoniensis alba, Dewdrop, Marjorie Daw, Decorus, Rubella, Argentea Guttata, Bertha, Fair Rosamond and Jessie. Also Tuberous and Rex Begonias.

Begonias-Leopard__._.

Ageratum Azureum

Acharanthus - red and var.

Cal. Holly

Centaurias-White and Yeliow -

Cineraria

Coleus

Vershaffeltii, Golden bedder, Trailing coleus.

Coleus in varieties.

Carnations

2 in. pots, per dozen $75 \mathrm{c}_{-}$

Cyperus - Alternafolia___._____ Cyclamen-White, red and white with red center.

Cyclamen

Cyclamen

Chrysanthemums - extra fine strains _._.__- per dozen $75 \mathrm{c}$

Daisies____

Geraniums

Varietics: Single white, double white, single pink, double pink, single salmon, double cherry red, single and double dark red Southey-single scarlet with white center. Mrs. E. H. Trego, semi-double scarlet.

John Forbes, Grants and others.

Ivys - Chas. Turner, Baden Powel, Joan of Are, and Mrs. Day Sweet Scented in variety Mary Washingtons, (Pelargoniums)
eliotrope-Varieties: Black Prince, Climbing Black Prince

Heliotrope-Varieties: Black Prince, Climbing Black Prince
Florence Nightingale, Snow Wreath in 4 in. pots

Matricarea inodora

Oleander--Pink, yellow and white

Pansies-Best strains, large flower, per dozen $25 \mathrm{c}$.

Pampas Grass

Petunias, fine double white per dozen $25 \mathrm{c}$.

Primula, Chinese 
Sweet Sultana
Solanum Cap, (Jerusalem cherry)
Verbenas.
Veronica-Maritima 4 in. pots
Violets-Tarieties: Princess, California, Double purple and
Swanley's white.

\section{BULBS.}

Agapanthus Umbellata (blue lily of the Nile)

Allium Grandiflorum (.._._._._._._._._. per dozen 25c Amaryllis Belladonna

Amaryllis (red)

Begonias (tuberous rooted)

Begonias (tuberous rooted) -............-. per dozen $75 \mathrm{c}$

Varieties: Single and double crimson, single and double pink, single and double rose, single and double white, and single and double yellow.

Calla Lily_..._._._._._._. 10 each and $75 \mathrm{c}$ per dozen

Cannas $5 \mathrm{c}$ each, and $60 \mathrm{c}$ per dozen.

Stenckoff-finest crimson.

Ensetifolia-tallest red.

Caladium - Fancy leaved

Caladium Esculentum

Chinese Sacred Lily .................. per dozen $35 \mathrm{c}$

Cyclamen ............. in 4 in. pots

Dahlias - Large double dark red and clear yellow, 5 to $25 \mathrm{c}$

Easter Lily Bulbs

Freesias

$15 \mathrm{c}$ per dozen

Gladiclus-Peachblow

Hyacinths-White - Warcissus-Paper White-

Eac
.20
.25

Shamrock.

per dozen $25 \mathrm{c}$

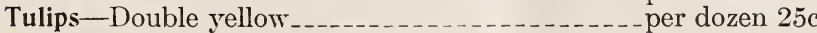
Tuberose Zephyranthes (Zephyr Flower)

\section{DRACÆNAS.}

Dracaena-Australis balled, 2 to $3 \mathrm{ft}$.

Dracaena-Indivisa in 5 gal. cans, 3 to $4 \mathrm{ft}$.

Dracaena-Indivisa $2 \mathrm{ft}$. in pots

\section{POTTED PALMS.}

Cycas-Revoluta, 8 to 12 leares

Cycas-Revoluta, 5 to 8 leaves 
Latania-Bourbonica 5 in. pots

Each

Phoenix-Canariensis 6 in. pots .40

Rhapis Flabeliformis in boxes, $2 \mathrm{ft}$.

Rhapis Flabeliformis in boxes, $3 \mathrm{ft}$.

Seaforthia-Elegans $2 \mathrm{ft}$., 6 in. pots

Chamerops -Excelsa Each

Phoenix-Canariensis

12 to $24 \mathrm{in}$.

Phoenix-Cnaariensis 1 to $2 \mathrm{ft}$. .75

Phoenix-Canariensis 2 to $3 \mathrm{ft}$. 1.25

Phoenix-Canariensis 3 to $4 \mathrm{ft}$. 1.50

Washingtonia-Robusta 4 to $5 \mathrm{ft} .2 .00$

Washingtonia-Robusta 1 to $2 \mathrm{ft}$.

Washingtonia-Robusta 2 to $3 \mathrm{ft}$.

Washintonia-Filifera 3 to $4 \mathrm{ft}$.

Washingtonia-Filifera

\section{HEDGE PLANTS.}

Monterey Cypress-per $1000, \$ 12.00$

Euonymus-Japonica

California Privet.

California Privet, 18 in. from field, -2.50 per 100.

\section{BORDER PLANTS.}

Alternanthera

Daisy

Euonymus-Pulchellus

Santolene

Ageratum

\subsection{0}

7.00

Flat

of

100

1.50

2.00

2.00

\section{CLIMBING AND TRAILING PLANTS.}

Ampelopsis-Quinquefolia, (Virginia Creeper)

Ampelopsis Veitchi (Boston Ivy)

Antigonon-Leptopus Asparagus-Plumosus Nanus, Asparagus - Plumosus Nanus. pots Asparagus -Sprengeri Asparagus-Hanging Baskets... Australian Pea Vine._._. pots Bignonia-Grandiflora, Radicans, Tweediana and Venusta
Bougainvillea Sanderiana Bougainvillea Sanderiana
Bougainvillea Braziliensis 
Honeysuckle-Chinese Evergreen

- 4 in. pots .25

Jasmine-Gracellum, and Officinale (white flowered)

Jasmine-Revolutum_._._.

Moonvine - White Flowered

Mulenbeckia Complexia (Wire Vine)

Passion Vines, red

Passion Vines, pink

Plumbago-Capensis (blue plumbago )

Plumbago-Alba

Snail Vine

Swainsona alba

Smilax

4 in. pots

Solanum-Wendlandii -Went.

Solanum-Wendlandii._._._._._.

Solanum Rantonnetti (Red flowered)

Tecoma Mackenii (pink trumpet flower)

Tecoma Capensis (red trumpet flower)

Trumpet Creeper.

Vinca-"Periwinkle" green-leaved, and varigated.

Wistaria- (Blue)

Wistraia (Blue)

\section{FERNS.}

Adiantum-Maiden Hair Fern -..._._._._. $25 \mathrm{c}$ and $50 \mathrm{c}$ each Nephrolepis Exaltata-Sword Ferns $25 \mathrm{c}$ and $50 \mathrm{c}$ each Boston $25 \mathrm{c}$ to 1.00 each Piersonii. Elegantissima Brakes - Single-Fine Clumps

Ferns in hanging basket. 1.00 to 3.00 each

\section{SEEDLINGS.}

Cypress-Monterey 
BEDDING STOCK.

Daisy-Shasta

Snowball

English

Geranium-Gen. Grant, per 100 in 3 inch pots

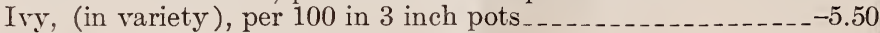

Verbenas in variety

Salvias -3 in. pots, $10 \mathrm{cts}$. each

Saxifragaria-(Strawberry Geraniums) - for Hanging Baskets 5c each

\section{OUR SPECIALTIES. Lippia Repens.}

California's finest Lawn Grass flat 18 by 18 inch

Will grow where all other lawns dry up-does not become a weed or run wild-once tried will never do without. (See fu: hor inside back cover.

\section{RHUBARB.}

CRIMSON WINTER RHUBARB Ask for prices by the 100 or 1000 .

This is one of Burbank's best productions-Tender, stringless, little water. Will yeild when other fail. (See inside back cover).

Victoria -

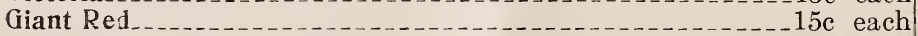

\section{FIELD GROWN ROSES.}

25 cents each or $\$ 2.50$ per dozen except where noted,

American Beauty, carmine.

Arch Duke Charles, red.

Black Prince, dark red.

Baby Rambler, crimson.

Beauty of Stapleford, rosy crimson.

Bride, white.

Bridesmaid, bright pink.

Bon Silene, crimson.

Burbank, red.

Catherine Mermet, light pink.

Cap. Christy, flesh pink.

Caroline Testout, pink.

Clothilde Soupert, white to pink.

Duchess de Brabant, light pink.

Duchess of Albany, deep rose.

Etoile de France, velvety red crimson.

Etoile de Lyon, yellow.

Golden Gate, shell pink.

Gen. R. E. Lee, orange yellow.

Gruss an Teplitz, red.

General Jaquiminot, red.

Helen Gould, rosey crimson.
Helen Gambier, orange yellow.

Isabella Sprunt, canary yellow.

Jules Finger, rosy scarlet.

Kaiserine, white.

Killarney, pale pink.

Laurette, cream.

LaFrance, pink.

Little Pet, white to pink.

Mrs. J. Laing, bright pink.

Madam F. Kruger, coppery yellow.

Madam de Wattville, yellow.

Madam de Berthod, deep golden yellow.

Magna Charta, deep pink.

Marie Van Houtte, pale yellow.

Malmaison, shell pink.

Maman Cochet, pink.

Meteor, red.

Madam Welche, amber yellow.

Madam de Vatry, pink.

Paquerett, white.

Paul Neyron, red.

Papa Gontier, carmine flushed crimson. 
President Carnot, light pink.

Perle des Jardins, deep yellow.

Perle de Or, coppery gold.

Rainbow, carmine striped with crimson.

Red Chateney, red.

Richmond, red.

$\triangle$ Southern Cal. Beauty, very large rosy pink.

Souv. of Wooton, red.

Snow Flake, white.

Sunset, tawny yellow.

Striped Henrietta, crimson and rose, $50 \mathrm{c}$. each.
Striped La France, white and pink shaded.

Souv, de Pierre Notting, creamy yellow.

Safrano, rich cream.

Uirich Bruner, crimson.

Vick's Caprice, rose striped with white.

Viscount Folkestone, pink and salmon.

White Malmaison, white.

White LaFrance, white.

White cochet, white with pink on outer petals.

\section{CLIMBING ROSES.}

Climbing Kaiserine, 'white.

Climbing Meteor, red.

Climbing La France, pink.

Climbing Perle, yellow.

Climbing Wooton, red.

Climbing Cecil Bruner, pink.

Crimson Rambler, crimson.

Caroline Goodrich, deep scarlet.

Cloth of Gold, yellow.

Double Cherokee, white.

Gold of Ophir, nasturtium yellow.

Henrietta, red.

Jas. Sprunt, dark red.
La Marque, white.

Mad. A. Carrier, flesh pink.

Marechal Neil, yellow.

Mary Washington, white tinted pink.

Rive de Or, orange yellow or deep saffron.

W. A. Richardson, deep orange center lighter.

White Rambler, white.

$\checkmark$ White Macrophylla, white.

White Banksia, white.

\section{CUT FLOWERS.}

(By IMail or Express)

Carnations, per dozen $25 \mathrm{c}$

Roses, per dozen 15 to $30 \mathrm{c}$

Violets, per bunch $.15 \mathrm{e}$

All Kinds of Floral Work-Floral designs, baskets and bouquets made up; prices according to size. These are packed and shipped with special care to guard against frost and heat. By Express Only.

Send Cash With Order-And say what you want, and we will make up according to money sent, always trying to give the best satisfaction.

\section{WIRE BASKETS.}

Wire Baskets, 10 inches

Each

Wire Baskets, 12 inches $\$ .35$

Wire Baskets, 14 inches

Flower Pots, 4 inches, 2 for..

Flower Pots, 5 inches

Flower Pots, 6 inches

(10

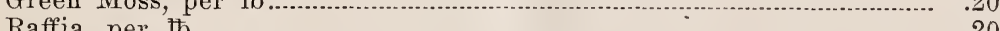

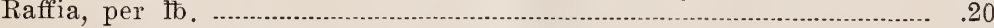

Transplanting Machines 


\section{NUMBER OF TREES ON AN ACRE.}

Number of Trees or Plants on an Acre at Given Distances Apart.

\section{IN SQUARES}

Distance 1 foot apart each way.

43560

" 2 feet apart

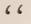

10890

" 3

" 


\section{INDEX}

Page

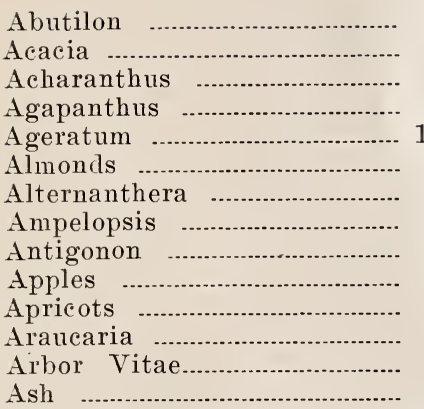

Asparagus Ferns...................... 10-1

Australian Pea Vine.............. 12

Begonia ...................................... 10-11

Bedding Stock.......................... 14

Bignonia

Blackberries

6

Blue Gums.

$7-13$

Border Plants.

12

Bougainvillea

Boxelder

Brachychitons (Sterculia)....

Banana

Bird of Paradise

Bridal Wreath.

Bulbs

Cal. Privet

Caladium

Calla Lillies

Crape Myrtle.

Page

10

Crytomera

11

$10-12$

6

12

12

12

4

4

Currants

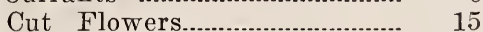

Cypress ........................................ 8

Cypress-Hedge Plants..........- 12

Cypress Seedlings-Flats...... 13

Cyperus ................................... 10

Daisies ......................................10-12-14

Deciduous Shrubs..................... 9

Dediduous Trees........................ 9

Dsutzia ........................................ 9

Dewberry ................................... 5

Dracaenas _................................. 11

Elm ............................................... 9

English Ivy................................. 12

Eucalyptus ................................ $7-13$

Eucalyptus, Seedlings, Flats 13

Euonymus ............................. 12

Euphorbia _............................... 9

Ferns .................................. 13

12 Ficus ......................................... $7-12$

9 Figs ......................................... 5

7 Floral Work............................... 15

10 Flowers, Cut............................. 15

9 Flower Pots................................ 15

9 Freesia ...................................... 11

11 Fuchsia .................................... 9

12 Geraniums .................................... 10-14

11 Gooseberry ................................ 6

11 Grafted Walnuts...................... 6

Camellia

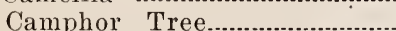

Catalpa

Carnations

$10-15$

Casuarina

Grapes

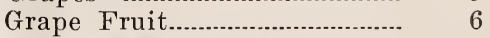

Grevillea-Robusta ................. 7

Guavas ..................................

7 Guavas-Weedlings-Flats...... 13

Cedrus deodora.

Centauria

Cherries

Chermoia

Chestnuts

Cineraria

Chrysanthemums

Citrus Fruits.

8 Hakea Eucalyptoides.............. T

10 Hedera .................................. 12

$4 \quad$ Hedge Plants............................. 12

7. Herbaceous Flowering Plants 10

6 Holly (Cal.).............................. 10

10 Honeysuckle ............................ 13

10 Helitrope ................................. 10

6 Hyacinths .................................. 11

12 Hydrangea ................................. 9

12 Hybiscus ........................................... 9

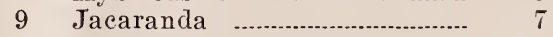

Climbing and Trailing Plants

Cocoloba

Coleus

Jasmine

Kum Quat.................................... 6

Coniferae

Lantannas 
Laurus Nobilis.

Lagunaria Pattersoni................

Lime

Live Oak.

Ligustrum

Lippia repens

Laurestinus

Lemon Verbena.

Lemons

Lilies

Lilac

Logan Berries

Loquats

Magnolia

Maple

Matricarea

Moonvine

Narcissus

Nectarines

Nuts

Oleander

Olives

Oranges

Ornamental Department........

Passion Vines.

Palms-Pot Plants.

Palms-Field Grown

Pampas grass.

Pansies

Pears

Peaches

Pecans

Pepper Tree

Persimmons

Petunias

Phoenix Palms...........................

Pines

Pines-Seedlings-Flats ........

Plumbago

Plums

Pittosporum

Poinsettia

Pomegranate

Pomelo-Grape Fruit

Primula

Privet
Page
Prune

Page

Quince

Raffia

Raspberry

Redwood

Rosedale Itybrid

Roses-Field Grown.

Rhubarb

Rubber Trees.

Seedlings in flats

Sterculia

Solanum

Smilax

Sultana

Saxifragaria

Salvia

Santolene

Sequoia

Snailvine

Snowball

Strawberry Plants.

Streptosolon

Swainsonia

Taxus erecta pyrimadalis.

Texas Umbrella.

Tecoma

Transplanters

Trumpet Creeper

Thuja

Thuyopsis

Tulips

Tulip poplar....

Vinca

Verbenas

Veronica

Violets

Vining Plants

Walnuts

Walnuts-Grafted

Walnuts-Eastern Black.......

Wire Vine

Wistaria

Wire Baskets.

Umbrella Tree

Zephyranthes
11-13

13

11

14

10-14

12

8

13

10

5

9

13

8 


\section{Crimson Winter Rhubarb.}

One of Luther Burbank's latest and best prodrctions. It meets every demand, both for home consumption and for market purposes.

It being a cross between one of the Australian varieties and the wild rhubarb of California:it is peculiarly adapted to our Southern Cal. ifornia climate. No home garden or market is complete without it.

Being in a growing condition trrelve months in the year it fills a long felt want in the home and in the market.

Stocks are tender and stringiless and of delicious flavor when cooked.

It is easy of culture, needing but little care; its returns are a hundred fold.

Our prices are so reasonable that the demands of all can be met.

Price 25 cents each, -2.50 per dozen. Special price by the 100 and 1000 .

\section{LIPPIA REPENS.}

This is a lawn covering material, forming a thick sward and takes much less water than grass or clover and stars green all the rear. Is not hard to eradicate when desired, ret will withstand drouth and abuse hetter than Bermuda grass, the home-maker's pest.

The sod is cut into pieces two inches square and set in the lawn 12 to 18 inches apart and Lippia Repens does the rest. It has a small whitish flower and somewhat resembles white clover at a distance.

It is a verbena, consequently easy to eradicate when desired.

It is sold in sods 18 by 18 inches and placed in shallow boxes for shipment. Price 75 cents per box. Special prices on large orders. 


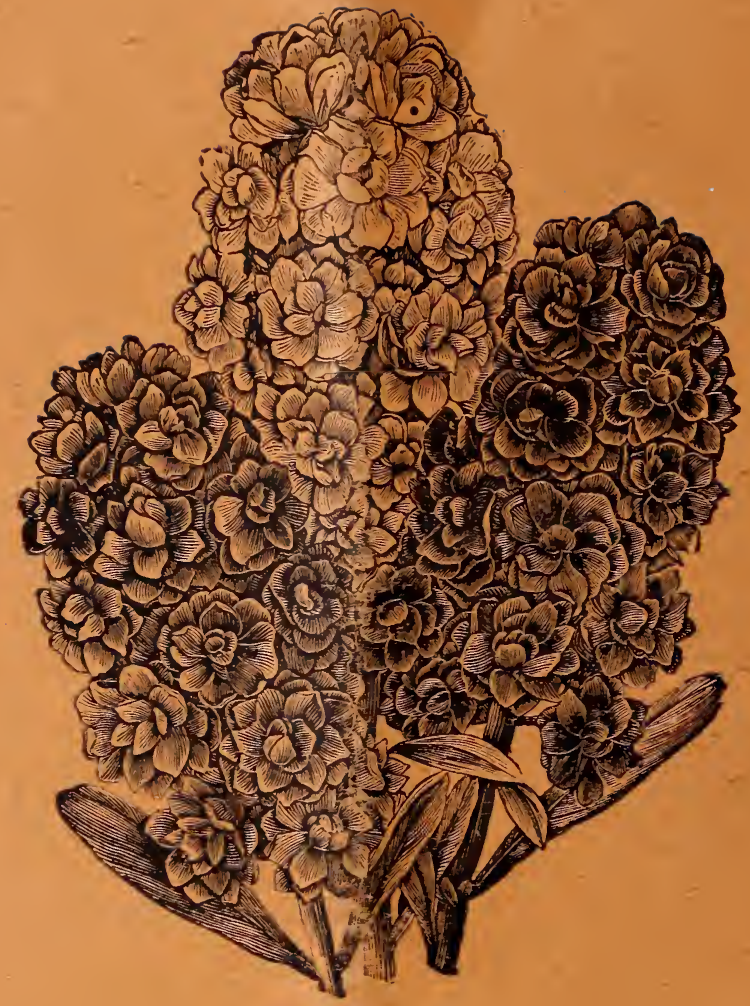

